Neurosurg Focus 17 (6):E9, 2004

\title{
Treatment of a chronic Scedosporium apiospermum vertebral osteomyelitis
}

\author{
Case report
}

\author{
John W. German, M.D., Susan M. Kellie, M.D., Manjunath P. Pai, Pharm.Dc., \\ and Paul T. Turner, M.D. \\ Divisions of Neurosurgery and Infectious Disease, and College of Pharmacy, University of New \\ Mexico, Albuquerque, New Mexico
}

\begin{abstract}
Scedosporium apiospermum is a rare cause of fungal vertebral osteomyelitis that may result in chronic infection requiring multiple surgical interventions and long-term medical therapy. This case is the seventh one reported in the literature and is the first to include salvage surgery of a previous major spinal reconstruction. This report is also the first to describe the use of the new antifungal agent voriconazole. In treating this case of chronic vertebral osteomyelitis, several principles are emphasized from both the surgical and medical perspectives. From a surgical perspective, the use of salvage surgery, temporary avoidance of spinal instrumentation, and an appropriate choice of graft materials are emphasized. From a medical perspective, confirmation of the diagnosis, the need for long-term antifungal therapy, the need for long-term patient compliance, and the use of the new antifungal agent voriconazole are emphasized. Application of these principles has led to an adequate 2-year outcome.
\end{abstract}

\section{KEY Words • fungus • osteomyelitis • Pseudallescheria boydii • \\ Scedosporium apiospermum • vertebral body • voriconazole}

Scedosporium apiospermum and Sc. prolificans are the two organisms responsible for scedosporiosis. ${ }^{19,20}$ The recognition of these rare human fungal pathogens is important because both are resistant to a variety of antifungal agents. The microorganism Sc. apiospermum is the anamorphic or asexual form of the ubiquitous saprophytic fungus Pseudallescheria boydii. Previously these organisms have been referred to as Monosporium apiospermum, P. sheari, Allescheria boydii, and Petriellidium boydii. ${ }^{19}$

The most common clinical manifestations of Sc. apiospermum infection are chronic cutaneous mycetoma, also known as "Madura foot," and pulmonary infection., ${ }^{6,19,20}$ The latter often occurs in the setting of previous tuberculosis. ${ }^{6,8,11}$ This association may confound diagnosis and delay appropriate treatment. Other infections associated with this organism include sinusitis, arthritis, and extremity osteomyelitis. ${ }^{20}$ Uncharacteristic sites of infection include the central nervous system and the spine. . $^{-6,8,11,12,20}$

Six cases of Sc. apiospermum vertebral osteomyelitis have been described in the literature. ${ }^{4-6,8,11,12}$ These cases are challenging in that they may evolve into a chronic, in-

\footnotetext{
Abbreviations used in this paper: $\mathrm{CRP}=\mathrm{C}$-reactive protein; $\mathrm{CT}=$ computerized tomography; ESR = erythrocyte sedimentation rate; TLSO $=$ thoracolumbosacral orthosis.
}

dolent infection that fails to respond to traditional antifungal agents. Management requires a multidisciplinary approach featuring surgical intervention for debridement, diagnosis, and spinal stabilization to preserve neurological function and/or spinal stability.

In this report we describe the clinical features, diagnosis, and management of the disease in a patient with a chronic, previously treated thoracolumbar osteomyelitis from an Sc. apiospermum infection.

\section{CASE REPORT}

History. This 44-year-old immunocompetent man with a long-standing history of intravenous drug abuse and known Sc. apiospermum osteomyelitis of the lumbar spine presented for further evaluation of increasing upper lumbar pain. Twenty-two months before the current presentation, he had undergone an L2-3 laminotomy for drainage of an epidural abscess, and the results of intraoperative cultures confirmed the diagnosis. He received intravenous itraconazole for several months and continued to take oral itraconazole (300 mg twice daily) until 6 weeks before presentation. At that time the drug was discontinued in anticipation of a biopsy procedure. Sixteen months before the latest presentation he had undergone a left retroperitoneal approach for an L-2 corpectomy with 
interbody carbon fiber cage reconstruction and L1-3 ventral instrumentation. He tolerated this procedure well and had good relief of his back pain until 4 months before presentation, when progressive back pain developed with upper lumbar radicular pain and paresthesia.

Examination. On presentation, the patient reported fever, back pain, upper lumbar radicular pain and paresthesia, urinary retention, and an inability to walk for the previous 2 weeks. Physical examination revealed a malnourished middle-aged man with significant spinal guarding and multiple upper-extremity subcutaneous abscesses. Neurological examination revealed weakness of the iliopsoas (Medical Research Council Score 3/5), quadriceps $(4-/ 5)$, tibialis anterior $(4+/ 5)$, and gastrocnemius $(4+/ 5)$ muscles; decreased sensation of light touch and pinprick in the L-1, L-2, and L-3 dermatomes; hypoactive reflexes at the quadriceps (1/4) and gastrocnemius (1/4) muscles; and downgoing toes bilaterally.

Neuroimaging Findings. A CT scan of the lumbar spine revealed a destructive process involving the vertebral bodies of L-1, L-2, and L-3 (Fig. 1) and large bilateral psoas abscesses (Fig. 2). The previously placed spinal instrumentation appeared loose with partial pullout of the upper screws.

Microbiological Findings. A preoperative CT-guided biopsy procedure confirmed the presence of a mixed Sc. apiospermum and Staphylococcus aureus osteomyelitis for which the patient received oral voriconazole (200 mg every 12 hours) and intravenous nafcillin ( 2 g every 4 hours), respectively.

Combined Medical and Surgical Management. Surgical management was initially planned as three separate staged procedures. A period of observed, inpatient bed rest was planned to help optimize medical management and to determine when an instrumented fusion for stabilization should be performed. An effort was made to maximize the amount of time the patient could be treated without spinal instrumentation. An inadequate ventral reconstruction was achieved at Stage 2 and required an unplanned revision. For the purpose of description, six stages of management are described as follows.

Stage 1: Instrumentation Removal. The patient underwent a standard retroperitoneal approach to the thoracolumbar spine through the previous incision on the left flank. On entering the retroperitoneal space, approximately $1700 \mathrm{ml}$ of free-flowing pus was evacuated and sent for culture. Further dissection proved difficult because of significant scarring of the retroperitoneum that limited the extent of the exposure. The previously placed ventral plate and screws were found to be mobile and were removed. The previously placed carbon fiber cage was also mobilized and removed. The previous corpectomy site was debrided as well as possible and the new trough was measured to accept a tricortical iliac crest bone graft that would be harvested at the beginning of Stage 2. Three 10$\mathrm{mm}$ Jackson-Pratt drains were placed into the retroperitoneal space close to the corpectomy site. Subsequently, an inflow and outflow irrigation system was established through the drains (Fig. 3). Intraoperatively obtained cultures grew $S$. aureus and Sc. apiospermum and the patient continued to receive intravenous nafcillin and oral voriconazole. He tolerated the procedure well, was kept on strict bed rest, and the inflow and outflow drains remained in place over the next 7 days.

Stage 2: Attempted Ventral Reconstruction. Seven days after undergoing Stage 1 treatment, the patient was returned to the operating room. He was placed supine, a large tricortical iliac crest graft was harvested, and the incision was closed. The patient was then placed in the left lateral decubitus position and a repeated retroperitoneal approach to the thoracolumbar spine was made through the existing left flank incision. The inflow and outflow drains were removed and the recently debrided trough was identified. Exposure of the graft site remained severely limited by an inflamed, enlarged psoas muscle. This precluded safe mobilization of the psoas away from the posterior vertebral bodies. The recently debrided corpectomy site was sized and the graft was fashioned. Caudal exposure of the corpectomy site remained significantly limited. Although the defect could be palpated, direct observation of the caudal end could not be accomplished because of the inflamed psoas musculature. The tricortical iliac crest bone graft was eventually tamped into place with the aid of fluoroscopy to guide the caudal end.

Stage 3: Prolonged Bed Rest and Observation. Postoperatively the patient tolerated the procedure well and his condition continued to improve, with $4+/ 5$ psoas and quadriceps strength as well as improvement of his upper lumbar sensation. He remained on 10 weeks of strict bed

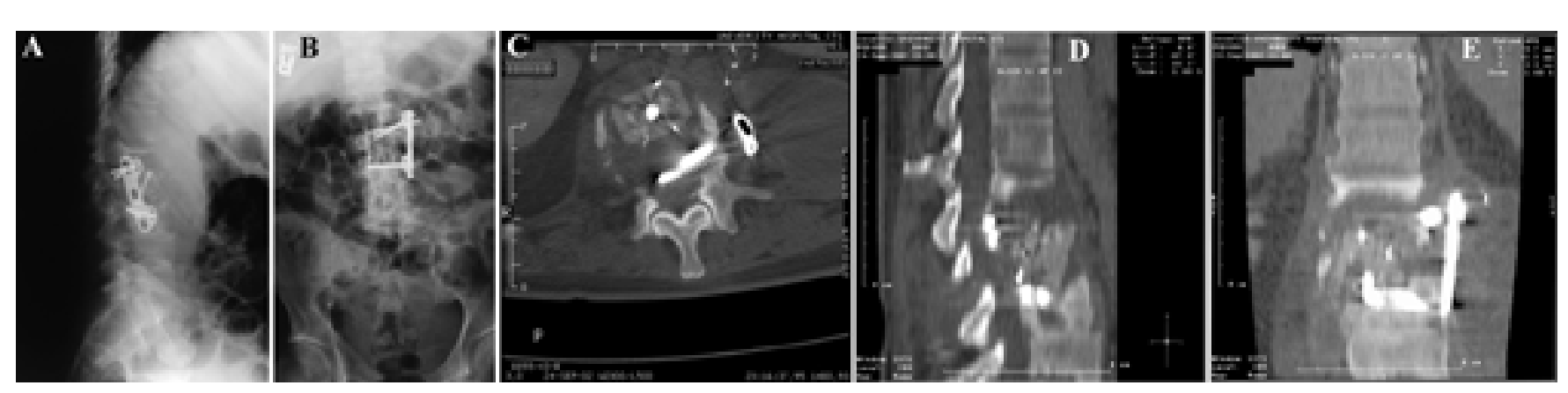

Fig. 1. A and B: Admission lateral and anteroposterior lumbar X-ray films showing the bone destruction in the L-1, L-2, and L-3 vertebral bodies. Note the displacement of the carbon fiber cage and partial pullout of the upper screws. C: Axial CT scan obtained at the level of the L-2 pedicle, demonstrating carbon fiber cage placement. D and E: Sagittal and coronal CT reconstructions. 


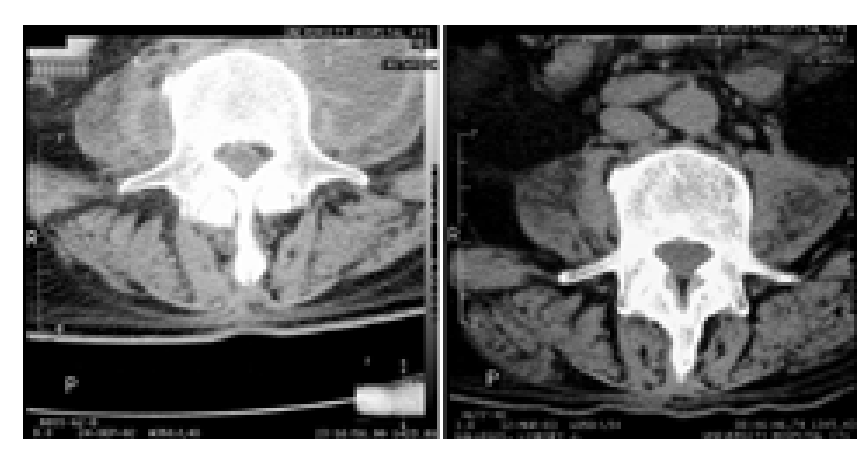

Fig. 2. Left: Admission axial CT scan obtained at the level of the L-4 pedicle, demonstrating the presence of bilateral psoas abscesses, which were larger on the left than the right. Right: Follow-up axial CT scan obtained at the level of the L-4 pedicle, demonstrating resolution of the psoas abscesses.

rest with prophylaxis for deep venous thrombosis. $\mathrm{Nu}$ tritional support was provided in the form of health shakes. During this time his antibiotic regimen continued with intravenous nafcillin and oral voriconazole. After 10 weeks he was fitted with a TLSO and mobilized with physical therapy. Standing X-ray films demonstrated dislodgement and posterolateral displacement of the iliac crest graft. The patient was discharged to a rehabilitation facility with instructions to wear the TLSO when upright. He continued on a regimen of oral Keflex (500 mg every 6 hours) and voriconazole (200 mg every 12 hours).

After discharge, the patient was seen every other week in outpatient follow-up review with serial spinal x-ray films. Initially he appeared to be doing well with minimal back pain. As anticipated, he subsequently reported increasingly severe back pain and intermittent leg pain. Serial upright thoracolumbar spinal x-ray films revealed progressive kyphosis and laterolisthesis (Fig. 4).

During this time diffuse lower-extremity edema developed in this patient. Results of lower-extremity Doppler evaluation were negative for deep venous thrombosis. We thought that his symptoms might represent a reaction to either the ibuprofen used for pain control or the Keflex, and both medications were accordingly discontinued. The patient was lost to follow-up care for a period of several weeks but subsequently presented again to the clinic after he was reincarcerated for violation of his probation by continued intravenous drug use. Given his increased lumbar pain and the radiologically confirmed progression of his deformity, we believed that surgical stabilization would be required.

Stage 4: Revision of Ventral Reconstruction. The patient was admitted to the hospital and received a preoperative bowel preparation. With the assistance a general surgeon, we subsequently performed a repeated left retroperitoneal approach to the thoracolumbar junction. Significant scarring of the retroperitoneum was again encountered but exposure was more easily accomplished because the psoas musculature had resumed a more normal size. The iliac crest graft was identified and found to be well adherent to the surrounding paraspinous tissue, but was nevertheless loose. The graft was removed and sent for culture, which proved negative. The corpectomy site was again debrided to obtain bleeding cancellous bone surfaces, and two locally harvested rib grafts were placed into the trough for interbody ventral support from T-12 to L-3 (Fig. 5). The patient tolerated the procedure well and was continued on the regimen of oral voriconazole (200 mg every 12 hours).

Stage 5: Dorsal Stabilization. Six days after undergoing Stage 4, the patient was returned to the operating room, where a posterior intertransverse process thoracolumbar fusion was performed with pedicle screw fixation from T-11 to L-4. The patient was then mobilized in a TLSO and discharged to the medical unit of the correctional facility. He continued to receive antibiotics including rifampin, voriconazole, and cephalexin.

Stage 6: Follow-Up Review. The patient continued to wear the TLSO for 3 months postoperatively, after which he was weaned from the brace. His recovery has continued to progress, with minimal back and leg pain. His last follow-up visit was approximately 25 months after initial presentation to our institution. Follow-up xray films have revealed no evidence of recurrent infection and they show maintenance of spinal alignment (Fig. 6).

\section{Voriconazole Levels}

Samples used to assess bone levels of voriconazole were obtained (Fugus Testing Lab, San Antonio, TX) at the initial debridement and removal of the carbon fiber

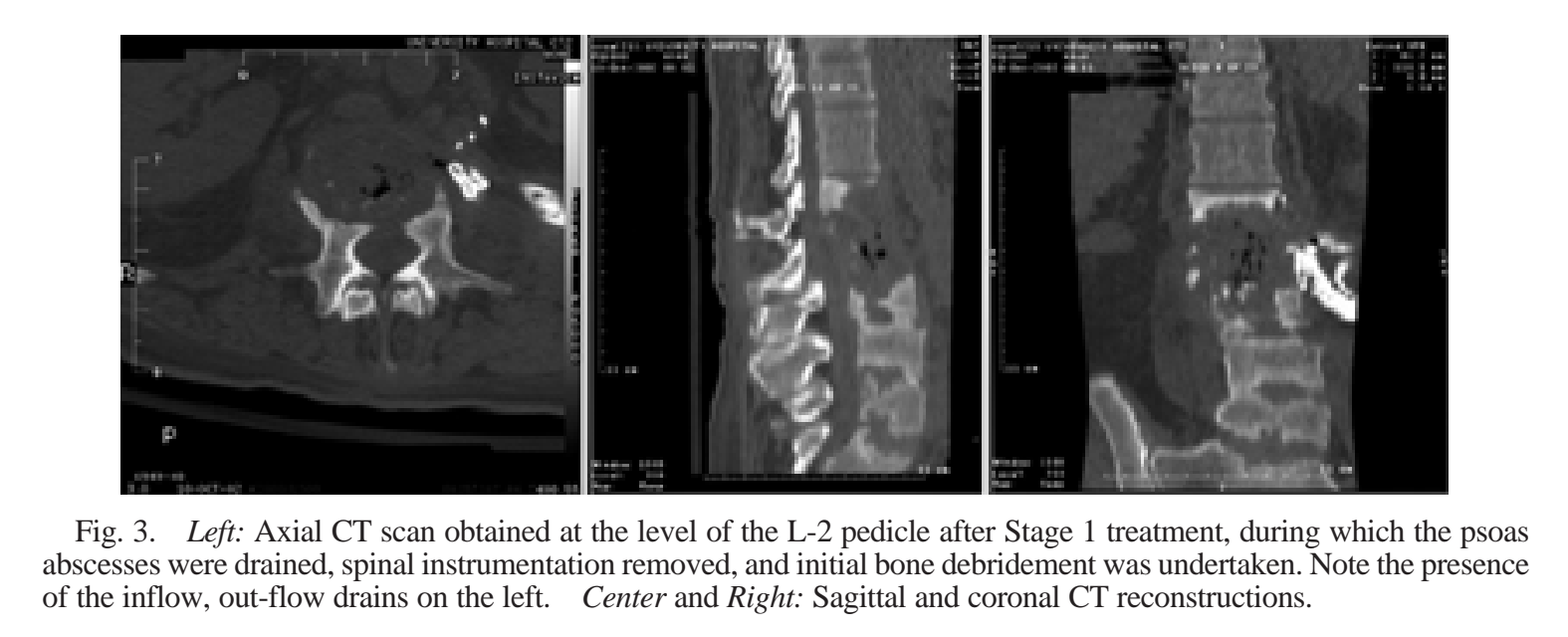



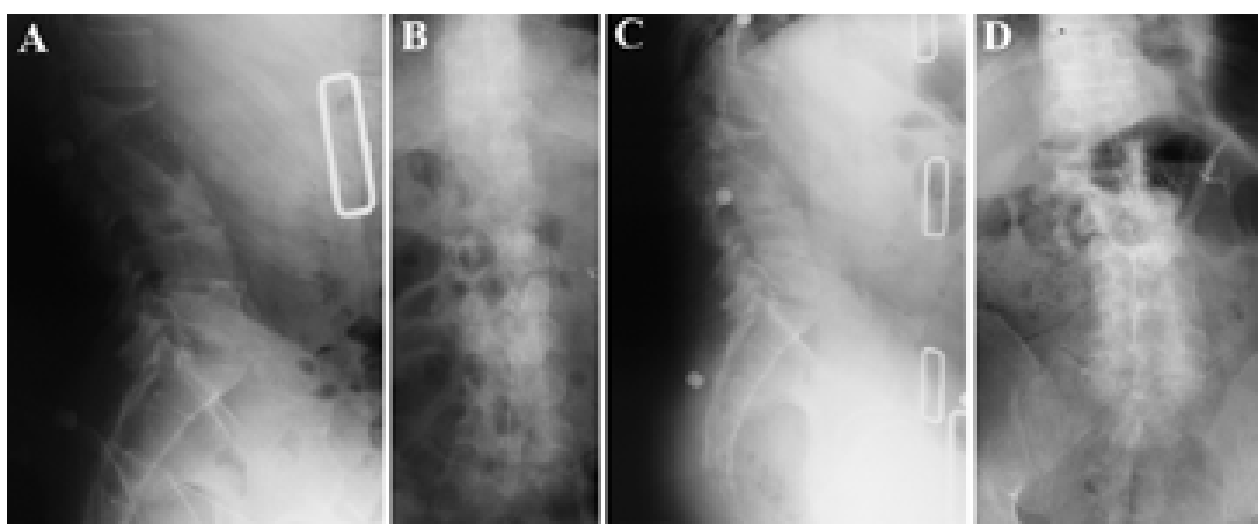

Fig. 4. A and B: Standing lateral and anteroposterior lumbar X-ray films obtained just after the period of bed rest concluded. C and D: Standing lateral and anteroposterior lumbar X-ray films obtained 3 months later. Note the progression of spinal deformity in both the coronal and sagittal planes.

cage after the patient had received the drug for 2 weeks. Briefly, two tiny cancellous bone fragments $(290 \mathrm{mg})$ were homogenized in a methanolic tetramethylethylenediamine buffer and extracted using solid-phase extraction. The eluate was analyzed using high-pressure liquid chromatography. The chromatogram was extremely clean and the voriconazole level was below the limit of detection $(<$ $0.2 \mathrm{mg} / \mathrm{ml}$ ). The patient had received a 200-mg dose of the drug 6 hours before surgery. Although a concurrent plasma sample was not obtained during the bone sample removal, previous blood samples documented a trough voriconazole concentration of $1 \mathrm{mg} / \mathrm{ml}$. Voriconazole therapy was continued despite the bone levels, given the improvements noted with the inflammatory markers as follows.

\section{Serological Monitoring}

Serial ESRs demonstrated normalization to $6 \mathrm{~mm}$ per hour after Stage 2. After the patient was discharged from the rehabilitation hospital, the ESR and CRP level increased to $27 \mathrm{~mm}$ per hour and $4.5 \mathrm{mg} / \mathrm{dl}$, respectively. The CRP level subsequently normalized to $0.5 \mathrm{mg} / \mathrm{dl} 3$ months after reincarceration and resumption of the antibiotic regimen.

\section{DISCUSSION}

According to the literature, $S c$. apiospermum is a rare cause of fungal vertebral osteomyelitis, with only six cases previously reported (Table 1). ${ }^{4-6,8,11,12}$ In our case, the patient presented with progressive spinal pain and weakness 16 months after an L-2 corpectomy and placement of spinal instrumentation. Progressive spinal pain is the most common presentation in patients with a fungal osteomyelitis. ${ }^{5,21}$ Most of the reported cases have been related to direct extension of a pulmonary infection, ${ }^{8}$ hematogenous spread, ${ }^{6,12}$ or direct inoculation. ${ }^{4,5}$ The infection has occurred in both immunocompromised ${ }^{4,6}$ and immunocompetent ${ }^{5,8,11,12}$ patients. With the growing population of immunocompromised patients from acquired immunodeficiency syndrome, organ transplantation, steroid use, total parenteral nutrition, and cancer treatment, these cases may continue to emerge. This will require some familiarity with the optimal multidisciplinary management of such infections on the part of spine surgeons and infectious disease specialists.

This case illustrates some of the difficulties in disease management in patients with fungal vertebral osteomyelitis in general and Sc. apiospermum in particular. In patients presenting with a new, destructive lesion of the spine, the diagnosis of a fungal infection is often delayed while treatment for either a presumed pyogenic or tuberculous process is initiated. ${ }^{5,6,8,11}$ It is only after failure of empirical medical treatment that the lesion is reexamined and the correct diagnosis established, often by pathological examination of surgically obtained tissue.
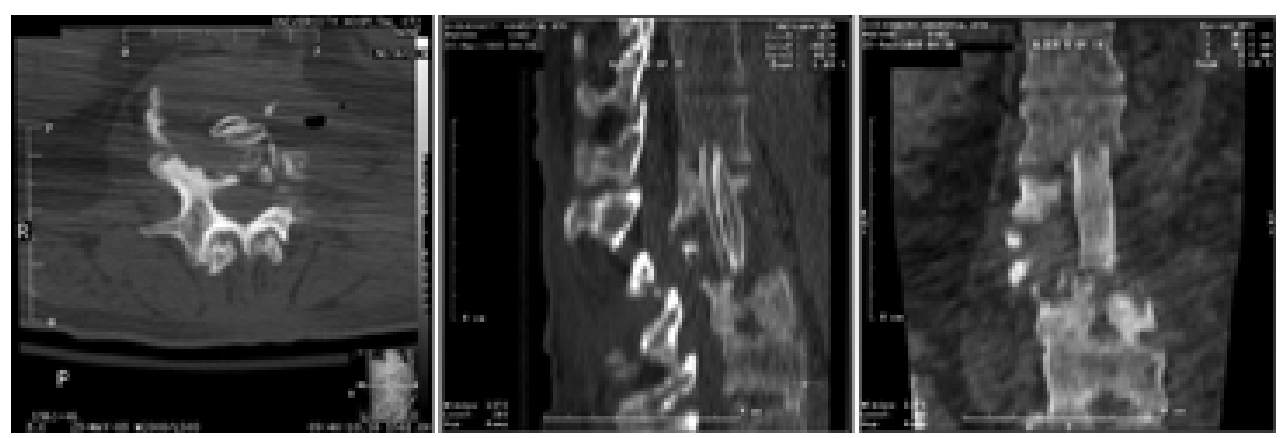

Fig. 5. Left: Axial CT scan obtained at the level of the L-2 pedicle after Stage 4 treatment, during which the previously attempted ventral reconstruction with iliac crest graft was removed. Note the rib grafts used in the revision as interbody struts. Center and Right: Sagittal and coronal CT reconstructions. 


\section{Treatment of chronic Sc. apiospermum vertebral osteomyelitis}

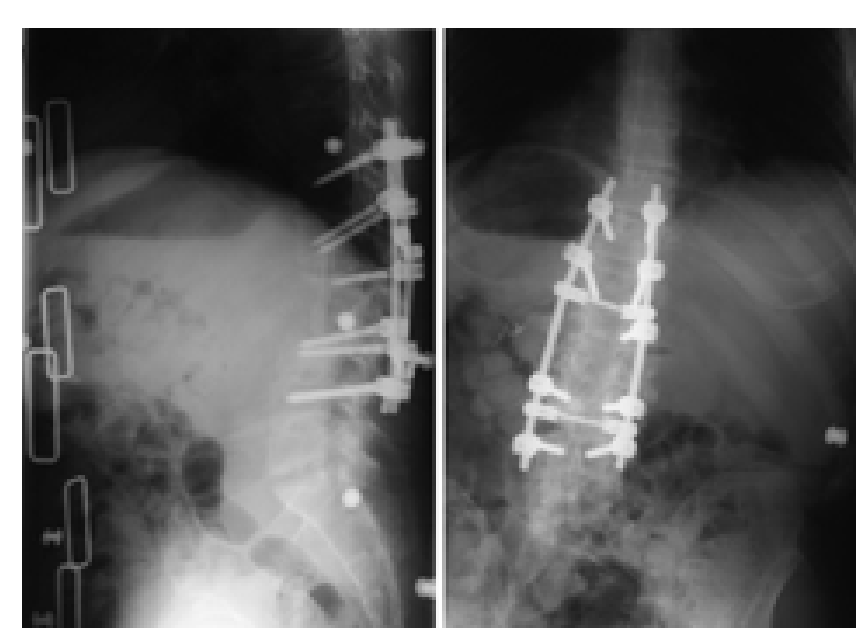

Fig. 6. Left and Right: Standing lateral and anteroposterior lumbar x-ray films obtained 3 months after the posterolateral fusion with T10-L4 pedicle screw fixation.

As demonstrated in this case report, the infection is often resistant to the traditional antifungal agents such as amphotericin B, miconazole, or itraconazole. ${ }^{19}$ This resistance leads to a chronic osteomyelitis with avascular necrosis of the bone and associated abscess formation. Treatment will eventually require a combined surgical and medical approach for optimal outcome.

\section{Defining Chronic Vertebral Osteomyelitis}

The distinction between an acute and a chronic osteomyelitis is an important but poorly defined concept in the management of vertebral osteomyelitis. The vast majority of clinical series include patients with pyogenic and/or tuberculous infections of relatively recent onset. ${ }^{1,3,15-17} \mathrm{~A}$ smaller number of reports have been published regarding the management of fungal osteomyelitis. ${ }^{5,21}$ In the majority of cases, initial management consisting of proper diagnosis, antimicrobial therapy, and bracing will be successful. ${ }^{1}$ If this initial management fails, resulting in spinal instability and/or neurological deficit, then surgical debridement and stabilization is considered. In this report we describe a clinical situation in which the infection progressed despite primary reconstruction. For the purpose of discussion, a working definition of a chronic vertebral osteomyelitis is as follows: an infection that fails to respond to the initial combined medical and surgical management.

\section{Surgical Management of Chronic Vertebral Osteomyelitis}

From a surgical perspective, this case highlights how the management of a chronic vertebral osteomyelitis and an acute infection differ. Three aspects of management are emphasized: use of salvage procedures, avoidance of spinal instrumentation, and choice of graft material.

\section{Salvage Procedures}

From the start we believed that three separate, staged salvage procedures would be required, with a planned period of bed rest during which spinal instrumentation would be avoided. In Stage 1 the infected instrumentation would be removed, an anterior debridement performed, and inflow, outflow drains placed. During the following week the diagnosis would be confirmed and antifungal therapy would be modified as needed. In Stage 2 an iliac crest autograft would be harvested and an interbody fusion without instrumentation performed. Stage 3 would consist of a prolonged period of bed rest during which the patient would be monitored clinically, radiographically, and serologically for evidence of disease progression. Stage 4 would consist of an instrumented posterolateral fusion that would be delayed as long as possible. The timing of Stage 4 would depend on the results of serial clinical examinations, radiographic studies, and laboratory tests for evidence of disease progression. This stage was eventually completed 9 months after the patient's initial presentation to our institution.

Salvage surgical procedures demand special attention and skill from the treating surgeon. Normally easily identifiable anatomical relationships are often poorly defined, scar tissue can limit mobilization of local anatomical structures, and exposure can be difficult to obtain. Such exposure can usually be accomplished by identifying the relatively normal anatomy above and below the previously instrumented levels. The instrumentation, bone graft, and edges of the previous corpectomy site are then defined. As our case illustrates, obtaining appropriate exposure for debridement and grafting may be extremely difficult in the setting of a chronic infection in which paraspinal abscess and inflamed paraspinous musculature limits retraction. Despite suboptimal placement of the interbody graft, the patient was kept on prolonged, observed bed rest for 10 weeks. During this time he improved, reporting less back pain, he demonstrated increased lowerextremity strength, and his ESR normalized.

\section{Instrumentation Avoidance}

In our opinion temporary avoidance of spinal instrumentation may be required, thus mandating a period of bed rest. Given the fact that this case represented a failed anterior reconstruction with continued active infection in the presence of spinal instrumentation, a repeated anterior debridement and reconstruction was required. In a review of the previously reported cases, we found two of six in which an initial posterior debridement failed. ${ }^{5,12}$ This is not surprising, because the infection often spares the posterior elements. This observation emphasizes the role of anterior debridement in aggressive or advanced spinal infections.

Whereas acute postoperative wound infections in the setting of posterior spinal instrumentation can be managed with soft-tissue debridement and the use of inflow, outflow drainage, ${ }^{10,14}$ it is unclear how best to manage spinal instrumentation associated with a chronic infection. Recent series of single-stage anterior debridement and fusion without instrumentation, ${ }^{1}$ single-stage anterior debridement and fusion with instrumentation, ${ }^{15}$ staged anterior debridement with sequential ${ }^{16}$ or delayed $^{3}$ posterior fusion with instrumentation have all been described and have yielded good results. It should again be emphasized that none of these series included either a chronic infection or a fungal osteomyelitis. No doubt this is related to the paucity of cases reported in the literature. By tradition, in the setting of chronic infection surgeons have removed or 
TABLE 1

Literature review of Sc. apiospermum vertebral osteomyelitis*

\begin{tabular}{|c|c|c|c|c|c|c|c|}
\hline \multirow[b]{2}{*}{ Authors \& Year } & \multirow[b]{2}{*}{$\begin{array}{l}\text { Age (yrs), } \\
\text { Sex }\end{array}$} & \multirow[b]{2}{*}{$\begin{array}{l}\text { Spinal } \\
\text { Levels }\end{array}$} & \multirow[b]{2}{*}{$\begin{array}{l}\text { Symptoms } \\
\text { \& Duration }\end{array}$} & \multirow[b]{2}{*}{$\begin{array}{l}\text { Immune } \\
\text { Status }\end{array}$} & \multirow[b]{2}{*}{$\begin{array}{l}\text { Source of } \\
\text { Infection }\end{array}$} & \multicolumn{2}{|c|}{ Treatment $\dagger$} \\
\hline & & & & & & Surgical & Medical \\
\hline Foster, et al., 1994 & $46, \mathrm{M}$ & $\mathrm{L} 2-4$ & $\begin{array}{l}\text { back pain, } \\
2 \text { mos }\end{array}$ & $\begin{array}{l}\text { compromised: } \\
\text { diabetes }\end{array}$ & $\begin{array}{l}\text { hematogenous } \\
\text { spread } \\
\text { from knees }\end{array}$ & $\begin{array}{l}\text { 1. L2-3 } \\
\text { posterolat } \\
\text { decompression }\end{array}$ & $\begin{array}{l}\text { 1. miconazole } \\
\text { 2. amphotericin B }\end{array}$ \\
\hline Hung, et al., 1994 & $69, \mathrm{M}$ & $\mathrm{T} 1-3$ & $\begin{array}{l}\text { back pain, } \\
9 \text { mos }\end{array}$ & competent & $\begin{array}{l}\text { direct extension of } \\
\text { pulmonary disease }\end{array}$ & $\begin{array}{l}\text { 1. } \mathrm{T} 1-3 \\
\text { laminectomy }\end{array}$ & 1. amphotericin B \\
\hline Gatto, et al., 1997 & $4, \mathrm{M}$ & L3-4 & $\begin{array}{l}\text { back pain, } \\
2 \text { wks }\end{array}$ & $\begin{array}{l}\text { compromised: } \\
\text { leukemia }\end{array}$ & $\begin{array}{l}\text { hematogenous spread } \\
\text { from hand }\end{array}$ & $\begin{array}{l}\text { 1. drainage of } \\
\text { L3-4 abscess }\end{array}$ & $\begin{array}{l}\text { 1. fluconazole } \\
\text { 2. itraconazole }\end{array}$ \\
\hline Frazier, et al., 2001 & $28, \mathrm{M}$ & $\mathrm{L} 2-3$ & $\begin{array}{l}\text { paraplegia after } \\
\text { traumatic } \\
\text { impalement }\end{array}$ & competent & direct inoculation & $\begin{array}{l}\text { 1. pst debridement } \\
\text { 1. ant corpectomy, } \\
\text { iliac strut graft, } \\
\text { pst instr }\end{array}$ & $\begin{array}{l}\text { 1. miconazole } \\
\text { 2. ketoconazole }\end{array}$ \\
\hline Lonser, et al., 2001 & $48, \mathrm{M}$ & T6-7 & $\begin{array}{l}\text { back pain, } \\
3 \text { mos }\end{array}$ & competent & $\begin{array}{l}\text { hematogenous spread } \\
\text { from injured hand }\end{array}$ & $\begin{array}{l}\text { 1. T6-7 } \\
\text { laminectomy } \\
\text { 1. T6-7 corpectomy } \\
\text { w/ fibula allograft } \\
\text { 2. T3-10 pst } \\
\text { fusion w/ instr }\end{array}$ & 1. itraconazole \\
\hline Levine, et al., 2002 & $52, \mathrm{M}$ & $\begin{array}{l}\text { T11-12, } \\
\text { L1-2 }\end{array}$ & $\begin{array}{l}\text { back pain, } \\
3 \text { mos }\end{array}$ & competent & unknown & $\begin{array}{l}\text { 1. T11-L2 } \\
\text { corpectomy w/ } \\
\text { tibia allograft } \\
\text { 2. T8-L3 pst fusion } \\
\text { w/ instr }\end{array}$ & 1. itraconazole \\
\hline present study & $44, \mathrm{M}$ & T12-L4 & $\begin{array}{l}\text { back pain, } \\
6 \text { mos }\end{array}$ & competent & $\begin{array}{l}\text { hematogenous } \\
\text { spread related } \\
\text { to iv drug abuse }\end{array}$ & $\begin{array}{l}\text { 1. L2-3 posterolat } \\
\text { decompression } \\
\text { 1. L-2 corpectomy } \\
\text { w/ carbon fiber cage } \\
\text { 1. removal of carbon } \\
\text { fiber cage } \\
\text { 2. attempted L1-3 } \\
\text { iliac crest autograft } \\
\text { 1. removal of L1-3 } \\
\text { iliac crest strut graft; } \\
\text { T10-L3 rib autograft } \\
\text { 2. T10-L4 pst fusion } \\
\text { w/ instr }\end{array}$ & $\begin{array}{l}\text { 1. itraconazole } \\
\text { 1. itraconazole } \\
\text { 1. voriconazole }\end{array}$ \\
\hline
\end{tabular}

* Ant = anterior; instr = instrumentation; iv = intravenous; pst = posterior.

$\dagger$ Numbers refer to stages of surgical intervention and corresponding medical treatments.

avoided placement of any material that may be considered foreign. In the setting of a chronic vertebral osteomyelitis, it is our belief that this basic tenet of surgery should be observed.

In our case this provided a 9-month time window during which the patient received medical therapy to optimize suppression of the infection. While his back pain and spinal deformity progressed over this time, he was temporarily lost to follow-up care. There is little doubt that shortly after discharge from the hospital the patient was noncompliant with his antibiotic regimen, as evidenced by a rise in both the CRP level and ESR. After reincarceration, the patient was again forced into compliance with his medical regimen, which resulted in a decrease in the CRP level. At this time it was believed that the patient should undergo revision of the anterior graft, followed by a staged posterolateral fusion with instrumentation.

\section{Graft Choice}

In the setting of a chronic infection, the spine surgeon should pay particular attention to the choice of reconstructive materials. It is believed that materials that may be considered foreign should be avoided. Such foreign materials would include carbon fiber (as previously used in our case), titanium, and allograft. Although the use of titanium cages and structural allografts has been reported to yield good outcomes in the literature, it should again be noted that these reports are limited to the management of pyogenic and tuberculous vertebral osteomyelitis of presumed recent onset. Good results have been reported in the literature for iliac crest or rib autograft in a variety of infections. ${ }^{1,5}$ Finally, use of a vascularized bone graft may be considered in cases of chronic osteomyelitis. ${ }^{2,13}$ These techniques are most commonly used in the thoracic spine and may be difficult to apply at the thoracolumbar junction.

\section{Medical Management of Chronic Vertebral Osteomyelitis}

From a medical perspective, the management of a chronic vertebral osteomyelitis starts with the diagnosis of continued active infection. As in our case, this may require cessation of antifungal agents and performance of tissue biopsy sampling in a delayed fashion. If results of the initial biopsy procedure are negative then repeated 
biopsy sampling, either CT-guided or open, will be required. The diagnosis of Sc. apiospermum is dependent on the results of culture and not fungal staining to differentiate the organism fully from Aspergillus spp. ${ }^{19,20}$ Such differentiation is important because it may change the choice of antifungal agent. ${ }^{20}$ After the diagnosis has been confirmed, the medical management of the disease in these patients differs from that of an acute infection in three aspects: 1) the need for chronic suppression therapy; 2) the need for long-term patient compliance; and 3) the use of new antifungal agents.

\section{Long-Term Suppression Therapy}

Patients with chronic infections will often require longterm antimicrobial therapy to prevent a further exacerbation of the infection. This is particularly important in patients with fungal infections, for whom medical regimens often require months if not years for completion. Previously standard antifungal agents such as amphotericin $\mathrm{B}$ and miconazole have relatively high-risk side effect profiles. Significant side effects include renal failure with amphotericin B and cardiac arrhythmia for miconazole. The latter drug was taken off the US market because of adverse side effects. Given these concerns, long-term therapy requires close clinical, radiographic, and laboratory monitoring. This ensures identification of disease progression as well as any adverse side effects of treatment. Our patient remains on chronic suppression therapy with oral voriconazole.

\section{Patient Compliance}

This case illustrates well the issues of compliance in the intravenous drug abusing population. Difficulties involved in treating osteomyelitis in this patient population have been emphasized by others. ${ }^{17}$ Despite, or perhaps as a result of a chronic infection that led to progression of his spinal deformity, our patient continued to use intravenous heroin until his admission to the hospital. Once hospitalized, his compliance was ensured by maintaining bed rest for approximately 3 months. During this interval he received his medical regimen in a closely monitored, inpatient fashion. Nevertheless, within weeks of being released from the hospital, the patient violated his parole by using intravenous heroin, for which he was subsequently reincarcerated. During this interval, compliance with his antifungal agent regimen was not ensured. An increase in his ESR during this interval was indicative of disease progression. Once reincarcerated, our patient's compliance was reestablished and he remained on the medical ward of the correctional facility for the next 9 months before again being released.

\section{Use of a New Antifungal Agent}

Voriconazole is a new antifungal agent of the triazole family that is active against pathogenic mold and yeast, including rare pathogens such as Fusarium spp. and Sc. apiospermum. ${ }^{9}$ Voriconazole is not active in vitro against Sc. prolificans and the Zygomycetes. Nevertheless, combined use of voriconazole and caspofungin has been associated with a successful outcome in an immunocompetent child with Sc. prolificans osteomyelitis. ${ }^{18}$ The penetration of triazoles into bone have not been well characterized.
Limited data reveal that itraconazole, which has a volume of distribution similar to voriconazole, has good bone penetration. ${ }^{7}$ The failure to measure detectable bone concentrations of voriconazole in this case was not predictive of the outcome. Consequently, voriconazole was a useful therapeutic option in this case, given its good bioavailability when administered orally and its tolerability despite several months of therapy.

\section{CONCLUSIONS}

Chronic fungal vertebral osteomyelitis is a rare infection that may require a modified treatment approach. With aggressive combined medical and surgical treatment, an adequate clinical outcome is possible.

\section{References}

1. Cahill DW, Love LC, Rechtine GR: Pyogenic osteomyelitis of the spine in the elderly. J Neurosurg 74:878-886, 1991

2. Deen HG, Zimmerman RS, Lanza LA: Vascular pedicle rib graft in anterior transthoracic fusion procedures. Technical note. J Neurosurg Spine 90:155-158, 1999

3. Dimar JR, Carreon LY, Glassman SD, et al: Treatment of pyogenic vertebral osteomyelitis with anterior debridement and fusion followed by delayed posterior spinal fusion. Spine 29: 326-332, 2004

4. Foster MR, Friedenberg ZB, Passero F: Lumbar Petriellidium boydii osteomyelitis with a systemic presentation. J Spinal Disord 7:356-360, 1994

5. Frazier DD, Campbell DR, Garvey TA, et al: Fungal infections of the spine. Report of eleven patients with long-term followup. J Bone Joint Surg Am 83:560-565, 2001

6. Gatto J, Paterson D, Davis L, et al: Vertebral osteomyelitis due to Pseudallesheria boydii. Pathology 29:238-240, 1997

7. Grant SM, Clissold SP: Itraconazole. A review of its pharmacodynamic and pharmacokinetic properties, and therapeutic use in superficial and systemic mycoses. Drugs 37:310-344, 1989

8. Hung CC, Chang SC, Yang PC, et al: Invasive pulmonary pseudallescheriasis with direct invasion of the thoracic spine in an immunocompetent patient. Eur J Clin Microbiol Infect Dis 13:749-751, 1994

9. Johnson LB, Kauffman CA: Voriconazole: a new triazole antifungal agent. Clin Infect Dis 36:630-637, 2003

10. Levi AD, Dickman CA, Sonntag VK: Management of postoperative infections after spinal instrumentation. J Neurosurg 86: 975-980, 1997

11. Levine NB, Kurokawa R, Fichtenbaum CJ, et al: An immunocompetent patient with primary Scedosporium apiospermum vertebral osteomelitis. J Spinal Disord Tech 15:425-430, 2002

12. Lonser RR, Brodke DS, Dailey AT: Vertebral osteomyelitis secondary to Pseudallescheria boydii. J Spinal Disord 14: 361-364, 2001

13. Nakamura H, Yamano Y, Seki M, et al: Use of folded vascularized rib graft in anterior fusion after treatment of thoracic and upper lumbar lesions. Technical note. J Neurosurg Spine 94: 323-327, 2001

14. Picada R, Winter RB, Lonstein JE, et al: Postoperative deep wound infection in adults after posterior lumbosacral spine fusion with instrumentation: incidence and management. J Spinal Disord 13:42-45, 2000

15. Przybylski GJ, Sharan AD: Single-stage autogenous bone grafting and internal fixation in the surgical management of pyogenic discitis and vertebral osteomyelitis. J Neurosurg Spine 94:1-7, 2001

16. Safran O, Rand N, Kaplan L, et al: Sequential or simultaneous, 
same-day anterior decompression and posterior stabilization in the management of vertebral osteomyelitis of the lumbar spine. Spine 23:1885-1890, 1998

17. Schuster JM, Avellino AM, Mann FA, et al: Use of structural allografts in spinal osteomyelitis: a review of 47 cases. J Neurosurg Spine 93:8-14, 2000

18. Steinbach WJ, Schell WA, Miller JL, et al: Scedosporium prolificans osteomyelitis in an immunocompetent child treated with voriconazole and caspofungin, as well as locally applied polyhexamethylene biguanide. J Clin Microbiol 41: 3981-3985, 2003

19. Tadros TS, Workowski KA, Siegel RJ, et al: Pathology of Hyalohyphomycosis caused by Scedosporium apiospermum (Pseudallescheria boydii): an emerging mycosis. Hum Pathol 29:1266-1272, 1998
20. Travis LB, Roberts GD, Wilson WR: Clinical significance of Pseudallescheria boydii: a review of 10 years' experience. Mayo Clin Proc 60:531-537, 1985

21. Wrobel CJ, Chappell ET, Taylor W: Clinical presentation, radiological findings, and treatment results of coccidioidomycosis involving the spine: report on 23 cases. J Neurosurg Spine 95:33-39, 2001

Manuscript received October 15, 2004.

Accepted in final form November 5, 2004.

Address reprint requests to: John W. German, M.D., 1211 Union Avenue, \#200, Memphis, Tennessee 38018. email: jwgerman@ hotmail.com. 